\title{
Early QCD analyses with photons at CMS
}

\author{
Pasquale Musella* \\ LIP Laboratorio de instrumantação e Fisica Experimental de Particulas \\ Lisboa, Portugal \\ E-mail: pasquale.musella@cern.ch
}

\section{On behalf of the CMS Collaboration}

The measurement of inclusive photon production is a crucial step for the understanding of Standard Model Physics at the Large Hadron Collider (LHC) and an important prerequisite for many new physics searches. The identification of photons' experimental signatures and their discrimination against instrumental background due to hadronic activity is a challenging task in the severe LHC environment. A robust set of selection criteria for the identification of isolated photons at the LHC start-up using the CMS detector was developed. In this work, the performance of such criteria is studied using proton-proton collision events at $\sqrt{s}=7 \mathrm{TeV}$ corresponding to $74 \mathrm{nb}^{-1}$. Results obtained in data are compared with expectations from Monte Carlo simulation and good agreement is observed.

35th International Conference of High Energy Physics - ICHEP2010,

July 22-28, 2010

Paris France

${ }^{*}$ Speaker. 
Events containing one or two prompt photons are produced, in proton-proton collisions, via Standard Model (SM) processes. The study of these production mechanisms is an important test of the SM predictions and allows the background rate for many searches, such as $H \rightarrow \gamma \gamma$, SUSY and extra-dimensions, to be constrained.

Photons are also produced in the decay of hadrons inside jets and may mimic prompt production; this background, mostly from energetic $\pi^{0}$ and $\eta$ mesons, is reduced through the photon identification techniques.

During the 2010 run, the Large Hadron Collider (LHC) [1] at CERN collided beams of protons at a centre of mass energy of $\sqrt{s}=7 \mathrm{TeV}$. In this work, data corresponding to $74 \pm 7 \mathrm{nb}^{-1}$ were used to study photon identification observables in the Compact Muon Solenoid (CMS) experiment.

CMS is one of the four experiments currently operating at the LHC. The central feature of the apparatus is a superconducting solenoid, of $6 \mathrm{~m}$ internal diameter, providing a field of $3.8 \mathrm{~T}$. Within the field volume are the silicon pixel and strip tracker, the crystal electromagnetic calorimeter (ECAL) and the brass/scintillator hadron calorimeter (HCAL). The ECAL covers the pseudorapidity ${ }^{1}$ region $|\eta|<3.0$ and was designed to have excellent energy resolution and high granularity, making it a powerful instrument to measure photons with high precision at the LHC. The ECAL is divided in a barrel section EB $(|\eta|<1.479)$ and two endcaps EE $(1.479<|\eta|<3.0)$. The ECAL granularity is $\Delta \eta \times \Delta \phi=0.0174^{2}$ in EB and $0.0174^{2} \leq \Delta \eta \times \Delta \phi \leq 0.05^{2}$ in EE. CMS uses a two level trigger system (labelled L1 and HLT), each designed to provide a data reduction factor of $\sim 10^{3}$. A detailed description of the CMS detector can be found elsewhere [2].

In the CMS detector, photons are primarily reconstructed through the energy deposited in the ECAL. The presence of material in front of the detector causes the photons to convert into electronpositron pairs. The bending of the electron and positron trajectories due to the solenoidal field leads the energy deposits to be spread along the bending direction. The energy deposited in individual crystals is thus grouped to obtain objects called super-clusters (SC) [3, 4]. The clustered energy is corrected taking into account interactions in the material in front of the ECAL and electromagnetic shower containment. The photon candidate pseudorapidity is corrected for the position of the reconstructed interaction vertex.

The distributions of photon identification observables in data were compared to those from a sample of events generated with PYTHIA 6 [5], tune D6T [6] and for which full detector response was simulated with GEANT 4 [7]. Events were required to be selected by the high-level trigger algorithm HLT_Photon15_L1R, which requires the presence of a SC with transverse energy above $15 \mathrm{GeV}$. The reconstructed transverse energy of the photon candidates was required to be larger than $30 \mathrm{GeV}$. The presence of a reconstructed primary interaction vertex consistent with a $p p$ collision was required. Only SC reconstructed in the region covered by the silicon tracker detector $\left(\left|\eta_{S C}\right| \leq 2.5\right)$ were considered. The region between the barrel and the endcaps $\left(1.4442<\left|\eta_{S C}\right|<1.566\right)$ was excluded. Super-clusters containing anomalous ECAL signals were removed by imposing requirements on topology and signal timing [8]. The hadronic to electromagnetic energy ratio was required to be $H / E<0.05$ to reject showers with a substantial hadronic component. To enrich the sample in isolated photons and study their characteristics, additional

\footnotetext{
${ }^{1}$ The pseudorapidity $\eta$ is defined as $\eta=-\ln \tan (\theta / 2)$, where $\theta$ is the polar angle measured with respect to the counterclockwise beam direction.
} 
selection requirements were applied. The selection criteria and the cut values were adjusted on the basis of Monte Carlo (MC) simulation, in absence of a data-driven control sample, aiming to maximise the background rejection while keeping the signal efficiency constant as a function of $\eta$ and $E_{T}$. A robust selection intended to be used for commissioning and early analysis was obtained in this way. Photon candidates had to satisfy three isolation criteria: 1) Iso $_{\text {TRK }}<2 \mathrm{GeV} / c$, where Iso ${ }_{\mathrm{TRK}}$ is the sum of the $p_{\mathrm{T}}$ of tracks compatible with the photon vertex in an annulus $0.04<R<0.40$, excluding a rectangular strip of $\Delta \eta \times \Delta \phi=0.015 \times 0.400$ to exclude the photon's own energy if it converts into an $\mathrm{e}^{+} \mathrm{e}^{-}$pair; 2) Iso ECAL $_{2}<4.2 \mathrm{GeV}$, where Iso ECAL $_{\text {in }}$ is the transverse energy deposited in ECAL in an annulus $0.06<R<0.40$, excluding a rectangular strip of $\Delta \eta \times \Delta \phi=0.04 \times 0.40$; and 3) $\mathrm{IsO}_{\mathrm{HCAL}}<2.2 \mathrm{GeV}$, where Iso $_{\mathrm{HCAL}}$ is the transverse energy deposited in HCAL in an annulus $0.15<R<0.40$. The $\eta-\eta$ element of the $\eta-\phi$ covariance matrix, $\sigma_{i \eta i \eta}$, which provides an expression ${ }^{2}$ for the extent in $\eta$ of the $\mathrm{SC}$, was required to be smaller than 0.01 (0.03) for SCs in EB (EE). Furthermore, SCs were required not to match pixel hits consistent with a track from the interaction region. The trigger selection criteria were shown to be fully efficient for SC with transverse energy above $20 \mathrm{GeV}$ [9]. In MC simulations, the efficiency of all other selection criteria for isolated photons is roughly $90 \%$ (80\%) in EB (EE). The total number of photon candidates which passed the selection in the analyzed data sample is about $2.9 \times 10^{3}$. The signal fraction in the selected sample is predicted to vary between 0.4 and 1 .

For each variable, the MC distributions were normalised to the total number of candidates in data, fixing the relative normalisation of each MC component using the cross-section calculated by PYTHIA. The difference between this normalisation and the one predicted using the total integrated luminosity and the total PYTHIA cross-section varies case-by-case and is $\lesssim 15 \%$. In each figure, the contribution due to isolated photons was separated from the one due to other sources. To compare the distribution of the variables entering the selection, all cuts except the one involving the variable under comparison were applied, obtaining the so-called $N-1$ distributions. Figure 1 shows the $N-1$ distributions for each isolation variable separately in the EB and EE regions. In general the agreement of the simulation with the data is satisfactory. The $N-1$ plots for the $\sigma_{i \eta i \eta}$ variable are also shown in Figure 1. The signal contribution to the peak below $0.01(0.03)$ is clearly visible in the EB (EE) regions. Figure 2 shows the transverse energy spectrum of photon candidates in data with all selection criteria applied. The shape of the spectrum is observed to be well described by the simulation over a large energy range.

In conclusion, using the first $7 \mathrm{TeV}$ data recorded by the CMS experiment, a study of the observables relevant for the identification of isolated photons was performed. With a selection intended to enrich the sample in signal photons and suppress the background from hadronic events, the performance of photon reconstruction and identification was shown to be similar to that expected from simulation. The commissioning of these techniques is an essential prerequisite for the measurement of SM processes with final state photons and to the search for new phenomena.

\footnotetext{
${ }^{2}$ This variable is defined by $\sigma_{i \eta \eta}^{2}=(\delta \eta)^{2} \sum_{i} w_{i}\left(\eta_{i}-\bar{\eta}\right)^{2} / \sum_{i} w_{i}$, where $w_{i}=\max \left(0,4.7+\ln \left(E_{i} / E\right)\right) . E_{i}$ is the energy of the $i^{\text {th }}$ crystal in a group of $5 \times 5$ centred in the one with the highest energy, $\eta_{i}$ is the $\eta$ index of the $i^{\text {th }}$ crystal and $\delta \eta$ is the average $\mathrm{EB} / \mathrm{EE} \eta$ granularity and $E=\sum E_{i}$ and $\bar{\eta}=\sum E_{i} w_{i} / \sum w_{i}$.
} 

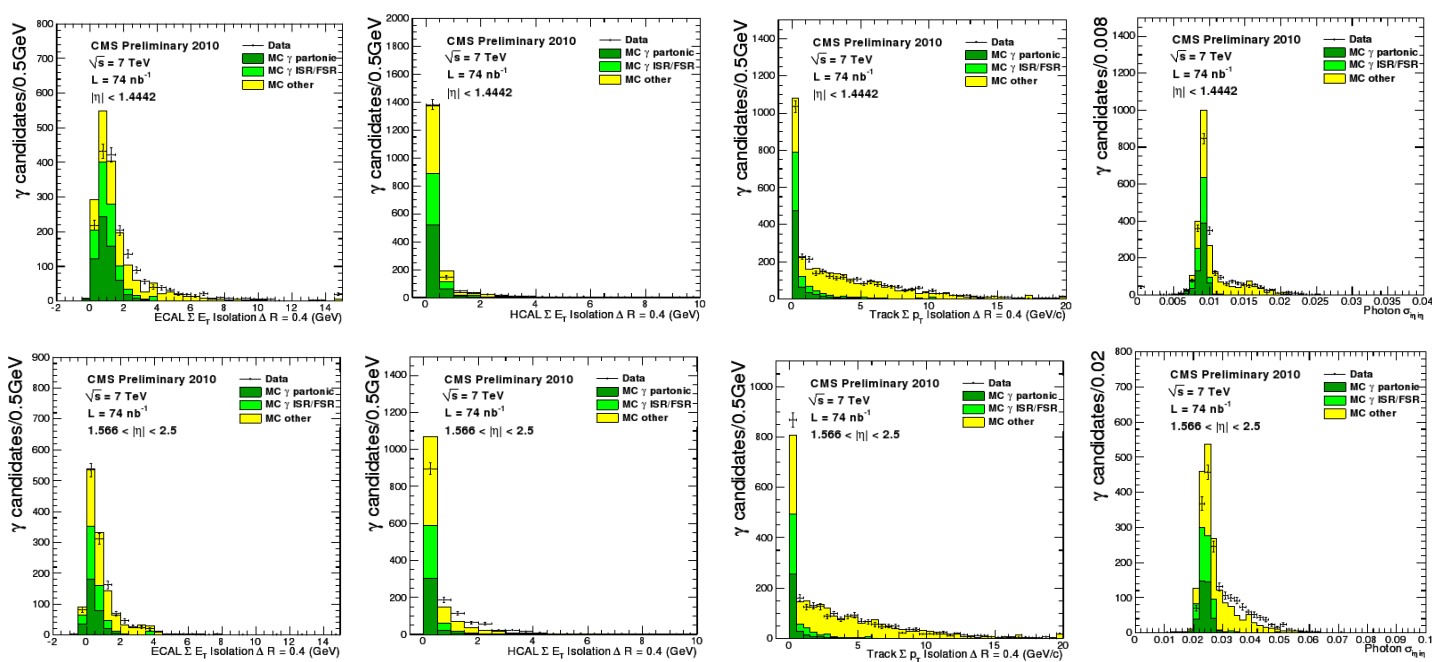

Figure 1: $N-1$ distribution of photon identification observables. In top row, the distribution of SCs in the EB region is shown, while in the bottom row the one of SCs from EE. From left to right, the distribution of

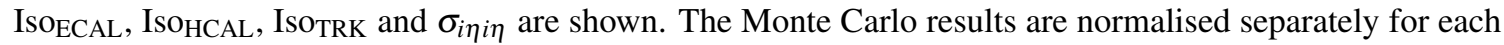
plot to the number of entries in the data histogram.
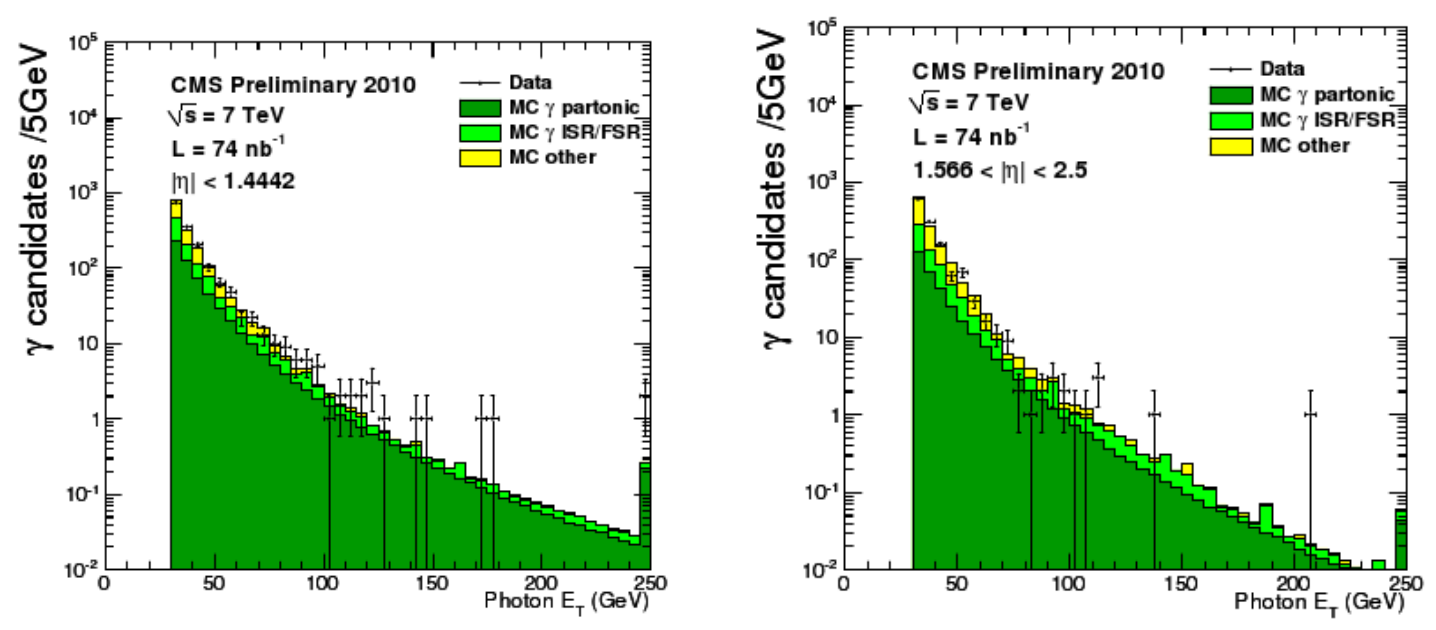

Figure 2: Transverse energy spectrum for photon candidates reconstructed in EB (left) and EE (right). The fraction of signal candidates increases with $E_{T}$. The Monte Carlo results are normalised separately for each plot to the number of entries in the data histogram. 


\section{References}

[1] L. Evans, (ed. ) and P. Bryant, (ed. ), "LHC Machine", JINST 3 (2008) S08001. doi:10.1088/1748-0221/3/08/s08001.

[2] CMS Collaboration, "The CMS experiment at the CERN LHC", JINST 3 (2008) S08004. doi:10.1088/1748-0221/3/08/S08004.

[3] CMS Collaboration, "CMS Physics Technical Design Report Volume I : Detector Performance and Software”, CMS Technical Design Report CMS-TDR-008-1 (2006).

[4] CMS Collaboration, "Photon reconstruction and identification at $\sqrt{s}=7 \mathrm{TeV}$ ", CMS Physics Analysis Summary EGM-10-005 (2010).

[5] T. Sjöstrand, S. Mrenna, and P. Z. Skands, "PYTHIA 6.4 Physics and Manual", JHEP 05 (2006) 026, arXiv:hep-ph/0603175. doi:10.1088/1126-6708/2006/05/026.

[6] M. G. Albrow et al., “Tevatron-for-LHC Report of the QCD Working Group”, arXiv:hep-ph/0610012.

[7] S. Agostinelli et al., "GEANT4 - a simulation toolkit", Nucl. Instr. Meth. A506 (2003) 250. doi:10.1016/S0168-9002(03)01368-8.

[8] CMS Collaboration, "Electromagnetic calorimeter commissioning and first results with $7 \mathrm{TeV}$ data", CMS Note CMS-NOTE-2010-012 (2010).

[9] C. Broutin, "Level-1 Electron and Photon Trigger Commissioning and Performance on $7 \mathrm{TeV}$ data", in Proceedings of the 35th Interntional Conference on High Energy Physics ICHEP. July, 2010. 